\title{
ISOTERMAS E CALOR ISOSTÉRICO DE SUBPRODUTOS DA UVA (VITIS VINIFERA)
}

\author{
C.F. GONÇALVES ${ }^{1}$, K.M. DEAMICI ${ }^{1}$, A.B. ARGENTA ${ }^{2}$, L.C. de OLIVEIRA ${ }^{3}$, G.S. \\ ROSA $^{4}$, E.G. de OLIVEIRA ${ }^{5 *}$ \\ ${ }^{1}$ Universidade Federal do Rio Grande, Programa de Pós-Graduação em Engenharia e Ciência \\ de Alimentos \\ ${ }^{2}$ Universidade Federal de Santa Maria, Programa de Pós-Graduação em Engenharia de \\ Processos \\ ${ }^{3}$ Universidade Federal do Pampa, Programa de Pós-Graduação em Engenharia \\ ${ }^{4}$ Universidade Federal do Pampa, Curso de Engenharia Química, \\ ${ }^{5}$ Universidade Federal do Rio Grande do Sul, Instituto de Ciência e Tecnologia de Alimentos, \\ Departamento de Tecnologia de Alimentos \\ *E-mail para contato: elizangela.oliveira@ufrgs.br
}

\begin{abstract}
RESUMO - Os subprodutos do processamento do vinho representam um problema de descarte devido ao volume gerado. O bagaço de uva se destaca pelo elevado teor de compostos fenólicos, fibras, além de ser fonte de compostos e nutrientes. O estudo das isotermas de sorção é importante para otimizar as condições de secagem do produto, assim como a sua estabilidade durante a armazenagem. O objetivo do trabalho foi avaliar as isotermas de dessorção do bagaço de uva das variedades Carbenet franc e Tannat nas temperaturas de 50, 60 e $70^{\circ} \mathrm{C}$. Os modelos de BET e GAB foram utilizados para ajuste dos dados experimentais e o calor isostérico de sorção foi determinado a partir da equação de Clausius-Clayperon. As isotermas apresentaram comportamento sigmoidal característico para este tipo de produto. Os modelos apresentaram bom ajuste aos dados experimentais, bons coeficientes de determinação e baixo valor para erro. A equação de Clausius-Clayperon se mostrou adequada para o cálculo do calor isostérico de sorção.
\end{abstract}

\section{INTRODUÇÃO}

Estudos relacionados ao plantio de uva são direcionados para se obter uma base da produção atual que esse setor apresenta no Brasil. Em termos de volume, foram colhidos 1.455.809 ton de uva em todo o território brasileiro, sendo o Rio Grande do Sul responsável por 840.225 ton, aproximadamente $58 \%$ da produção total de uvas colhidas em todo território brasileiro referente à safra de 2012 (Mello, 2013).

A constante produção de derivados da uva, principalmente de vinhos, gera uma quantidade de subprodutos (bagaço de uva) que pode chegar até $25 \%$ do beneficiamento total do produto. $\mathrm{O}$ bagaço de uva representa para as vinícolas um problema ambiental de descarte devido ao grande volume gerado, o qual é considerado fonte potencial de compostos e nutrientes podendo ser aproveitados para elaboração de novos produtos (Shieber et al., 2001). 
Dentre as alternativas para o aproveitamento desse subproduto destaca-se o processo de secagem, que envolve a retirada parcial de água do material através da transferência simultânea de calor do ar para o material e de água, por meio de fluxo de vapor, do material para o ar (Andrade et al., 2006). Neste processo, é de suma importância o conhecimento da relação de equilíbrio termodinâmico entre o sistema composto pelo material sólido e o ar (Goneli, 2008).

O estudo das isotermas de sorção, também denominadas isotermas de equilíbrio, permite caracterizar o equilíbrio termodinâmico, pois estas descrevem a relação de equilíbrio entre o teor de umidade de um material e a umidade relativa $(U R)$ do ambiente, a uma dada temperatura. A variação no teor de água no sólido ocorre até que a pressão de vapor do sólido e a pressão de vapor do ar circundante se tornem iguais. Assim, a umidade de equilíbrio $\left(X_{e}\right)$ do material pode ser atingida por acréscimo (adsorção - a partir do estado seco) ou perda (dessorção - a partir do estado úmido) de umidade do material em função da umidade relativa do meio (Bhandari et al., 2008; Marinos-Kouris et al., 2006).

Vários métodos vêm sendo utilizados para determinação das isotermas de sorção de alimentos e produtos agrícolas. O método gravimétrico, que envolve a utilização de soluções salinas saturadas ou soluções de ácido sulfúrico em diferentes concentrações para manter a umidade relativa constante, tem sido considerado preferível para obtenção das isotermas de sorção (Iguaz et al., 2007).

O conhecimento do calor isostérico em função da umidade de equilíbrio é essencial nos estudos de secagem, armazenamento de produtos agrícolas e capaz de estimar as necessidades energéticas do processo de secagem. Através do calor isostérico pode-se estimar a quantidade mínima de calor requerida para remover uma quantidade de água e obter-se algumas deduções sobre a microestrutura e as mudanças físicas que acontecem na superfície dos alimentos. $\mathrm{O}$ calor isostérico é uma propriedade termodinâmica de sorção de água crucial na análise do fenômeno de transferência de calor e de massa (CAMPOS et al., 2009; LIMA et al., 2008).

Sendo assim, o desenvolvimento do trabalho teve como objetivo avaliar as isotermas de dessorção do bagaço de uva fermentado das variedades Carbenet franc e Tannat, nas temperaturas de 50,60 e $70^{\circ} \mathrm{C}$, além de calcular o calor isostérico de dessorção.

\section{MATERIAS E MÉTODOS}

\subsection{Matéria-Prima}

O subproduto utilizado neste trabalho foi o bagaço de uva fermentado das variedades Carbenet franc e Tannat proveniente da safra 2011/2012, fornecido por uma Vinícola localizada na cidade de Bagé - RS. O subproduto foi armazenado em sacos plásticos e acondicionado à temperatura de $-18^{\circ} \mathrm{C}$ em freezer localizado no Laboratório de Engenharia de Alimentos da Universidade Federal do Pampa. E antes da realização dos experimentos as amostras foram descongeladas em refrigerador a aproximadamente $4^{\circ} \mathrm{C}$ por $24 \mathrm{~h}$.

\subsection{Isotermas de Sorção}


As amostras in natura foram imersas em solução de sorbato de potássio $20 \%$ (p/v) por um período de $60 \mathrm{~min}$, para evitar a proliferação de micro-organismos. Após, foram retiradas e colocadas em papel toalha por $15 \mathrm{~min}$, em temperatura ambiente, de forma a retirar o excesso de umidade do material. Posteriormente, as amostras foram caracterizadas quanto à umidade, a qual foi determinada pelo método da Association of Official Analytical Chemists (AOAC, 1995).

As isotermas foram realizadas através do método gravimétrico estático, em triplicata, com soluções ácidas em onze diferentes concentrações, garantindo assim uma ampla faixa de variação de umidade relativa. Foram utilizados recipientes de vidro com $7 \mathrm{~cm}$ de altura e 6 $\mathrm{cm}$ de diâmetro, hermeticamente fechados, garantindo assim uma atmosfera constante. No interior dos recipientes, além do suporte plástico para inserção da amostra, continham as soluções de ácido sulfúrico em diferentes concentrações, variando de 0,20 até $0,70 \mathrm{~kg} \cdot \mathrm{kg}^{-1}$. A massa inicial de amostra do bagaço de uva in natura utilizada foi de aproximadamente $3 \mathrm{~g}$.

O sistema, apresentado na Figura 1, permaneceu em estufa, nas temperaturas de 50, $60 \mathrm{e}$ $70{ }^{\circ} \mathrm{C}$ até que a condição de equilíbrio fosse atingida, sendo realizadas pesagens periódicas em balança analítica com precisão de 0,0001 .

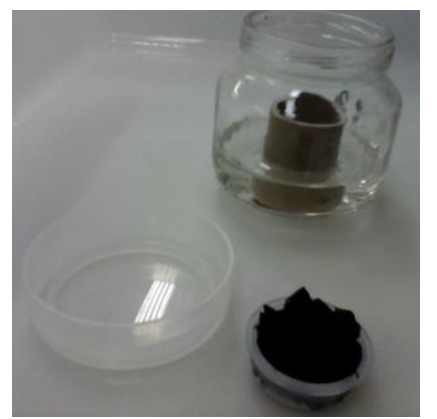

Figura 1 - Aparato para determinação das isotermas de sorção.

Com a condição de equilíbrio estabelecida, foi realizada a análise de umidade pelo método da estufa a $105^{\circ} \mathrm{C}$ por $24 \mathrm{~h}$, seguindo o método da AOAC (1995).

\subsection{Metodologia de Cálculo}

Para o ajuste das isotermas de dessorção foram aplicados os modelos de BET e GAB, Equações 1 e 2 respectivamente, possibilitando obter os valores da umidade de monocamada $\left(X_{m}\right)$ e o valor da umidade de equilíbrio $\left(X_{e}\right)$ bem como os demais parâmetros de cada modelo.

$$
\begin{aligned}
& X_{e}=\frac{X_{m} \cdot C_{B} \cdot U R}{(1-U R) \cdot\left(1-U R+C_{B}+U R\right)} \\
& X_{e}=\frac{X_{m} \cdot C_{G} \cdot K \cdot U R}{(1-K \cdot U R) \cdot\left(1-K \cdot U R+C_{G} \cdot K \cdot U R\right)}
\end{aligned}
$$

em que $\left(X_{e}\right)$ é a umidade de equilíbrio do material $\left(k g_{\text {água }} / \mathrm{kg}_{\text {sólio seco }}\right),\left(X_{m}\right)$ a umidade da monocamada do material ( $\left.k g_{\text {água }} / k_{\text {sólio seco }}\right),(U R)$ é a umidade relativa do ar e $C_{B}, C_{G}$ e $K$ 
são parâmetros associados às entalpias de sorção na monocamada e multicamada, respectivamente.

Os parâmetros das equações foram determinados através de análises de regressões não lineares, pelo método de quasi-Newton, através de um software estatístico. O coeficiente de determinação $\left(R^{2}\right)$, foi o critério de escolha para o melhor ajuste das equações avaliadas, para definir as isotermas de sorção de bagaço de uva. Além do $R^{2}$, também foi avaliado o valor da raiz quadrada do erro médio (RQEM).

Para determinação do calor isostérico de dessorção utilizou-se a Equação de ClausiusClayperon, Equação 3, a qual relaciona a mudança da umidade relativa do ar com a temperatura, onde plotando-se $\ln (U R)$ versus $1 / T$, para uma umidade específica, determina-se a inclinação da reta, que equivale a $-q_{s t} / R$. O calor isostérico total de sorção no alimento $\left(q_{t}\right)$ é definido como o calor isostérico líquido de sorção $\left(-q_{s t}\right)$ mais o calor de vaporização da água pura $\left(\Delta H_{v a p}\right)$, a uma dada temperatura, conforme Equação 4 (LEMUS, 2008):

$$
\begin{aligned}
& q_{s t}=-R \cdot \frac{\partial \ln (U R)}{\partial(1 / T)} \\
& q_{t}=q_{s t}+\Delta H_{v a p}
\end{aligned}
$$

em que $q_{s t}$ é o calor isostérico líquido de sorção $(\mathrm{kJ} / \mathrm{mol}), R$ é a constante universal dos gases (8,314 J/mol.K), $U R$ é a umidade relativa do ar, $T$ é a temperatura absoluta $(\mathrm{K}), q_{t}$ é o calor isostérico total de sorção $(\mathrm{kJ} / \mathrm{mol})$ e $\Delta H_{\text {vap }}$ é o calor de vaporização da água $(\mathrm{kJ} / \mathrm{mol})$.

\section{RESULTADOS E DISCUSSÃO}

A umidade inicial das amostras das variedades Cabernet franc e Tannat, determinada pelo método da AOAC (1995), resultou em $61,87 \pm 0,76 \%$ e $65,31 \pm 0,93 \%$ de umidade em base úmida, respectivamente.

A Figura 2 apresenta as isotermas de equilíbrio, que relacionam a umidade de equilíbrio $\left(X_{e}\right)$ das amostras com a umidade relativa do ar $(U R)$, determinadas para o bagaço de uva das variedades Cabernet franc e Tannat, respectivamente.
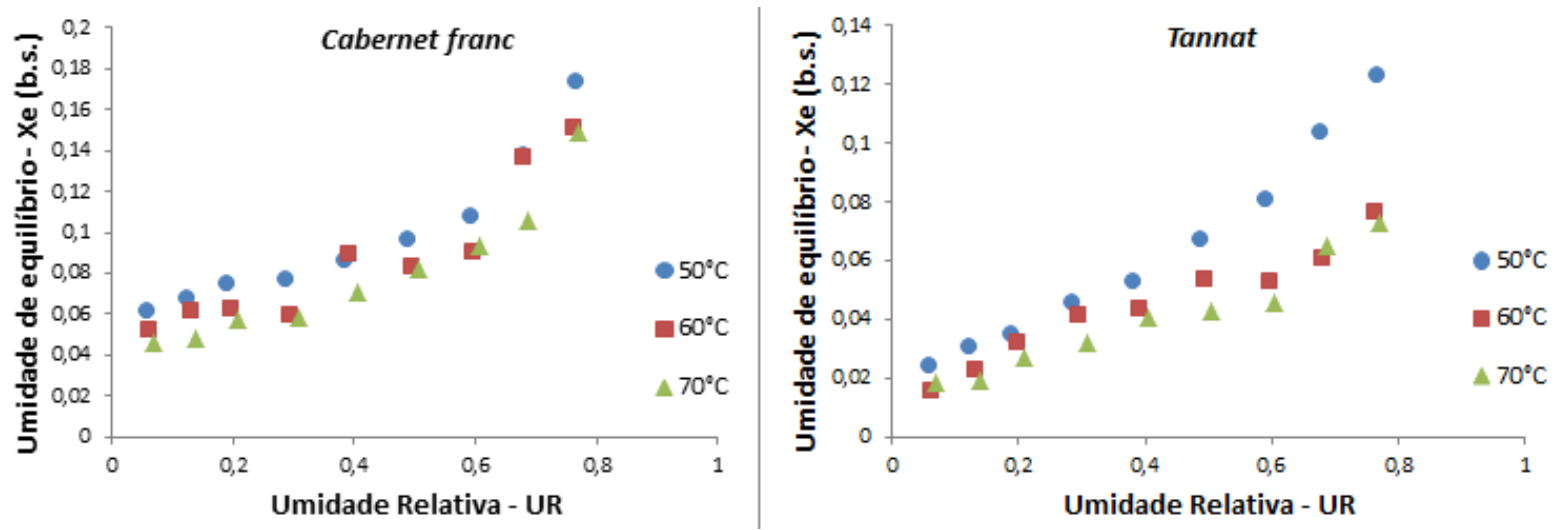

Figura 2 - Isotermas de equilíbrio para o bagaço de uva nas temperaturas estudadas. 
Observa-se que as curvas apresentaram comportamento sigmoidal típico para isotermas de alimentos. Pode-se observar que para um valor de $U R$ constante, o aumento da temperatura provocou redução na umidade de equilíbrio, como esperado. No estudo realizado por Lemus et al. (2008) pode-se observar o mesmo comportamento para determinação das isotermas de equilíbrio de alga vermelha Gracilaria, onde foi verificado que o conteúdo de umidade de equilíbrio aumentou a medida que a temperatura diminuiu.

Luz et al. (2006) observaram valores de umidade de equilíbrio para as isotermas de farelo de soja variando de 0,034 a 0,240 para $50{ }^{\circ} \mathrm{C}$, de 0,029 a 0,233 para $60{ }^{\circ} \mathrm{C}$ e 0,023 a 0,221 para $70{ }^{\circ} \mathrm{C}$. Pode-se verificar então, que com o aumento da temperatura do ar, a umidade de equilíbrio do farelo de soja sofre pequena redução no valor da umidade de equilíbrio.

No presente trabalho, pode-se ainda verificar que quando comparadas as duas variedades, Cabernet franc e Tannat, a segunda apresentou umidade de equilíbrio inferior para as três temperaturas estudadas. Isso se deve ao fato dessa amostra possuir menos sementes, o que facilita o processo de secagem e, consequentemente, de redução de umidade. A amostra da variedade Cabernet franc apresentava uma parcela de sementes em sua composição, dificultando a remoção de umidade.

Com os dados de isotermas obtidos, realizou-se uma regressão não linear através do método dos mínimos quadrados, utilizando os modelos de GAB e BET. Os resultados dos ajustes para as temperaturas estudadas estão apresentados na Tabela 1.

Tabela 1 - Parâmetros obtidos nos ajustes para cada modelo e temperatura de estudo.

\begin{tabular}{|c|c|c|c|c|c|c|c|}
\hline \multirow{4}{*}{$\begin{array}{c}\text { Modelos } \\
\text { Temperatura } \\
\left({ }^{\circ} \mathrm{C}\right)\end{array}$} & & \multicolumn{6}{|c|}{ Cabernet franc } \\
\hline & & \multicolumn{3}{|c|}{ GAB } & \multicolumn{3}{|c|}{ BET } \\
\hline & & 50 & 60 & 70 & 50 & 60 & 70 \\
\hline & $X_{m}$ & 0,057 & 0,050 & 0,041 & 0,046 & 0,041 & 0,035 \\
\hline \multirow[t]{2}{*}{ Parâmetros } & $C$ & 6242341,7 & 564924,7 & 99175139,9 & 6320979,1 & 1832407,8 & 6144189 \\
\hline & $\boldsymbol{K}$ & 0,865 & 0,882 & 0,922 & - & - & - \\
\hline $\mathbf{R}^{2}(\%)$ & & 99,2 & 96,6 & 97,6 & 92,4 & 92,7 & 94,6 \\
\hline \multirow[t]{2}{*}{ RQEM (\%) } & & 0,44 & 0,85 & 0,80 & 1,33 & 1,31 & 1,00 \\
\hline & & \multicolumn{6}{|c|}{ Tannat } \\
\hline Modelos & & \multicolumn{3}{|c|}{ GAB } & \multicolumn{3}{|c|}{ BET } \\
\hline \multirow[t]{2}{*}{$\begin{array}{c}\text { Temperatura } \\
\left({ }^{\circ} \mathbf{C}\right)\end{array}$} & & 50 & 60 & 70 & 50 & 60 & 70 \\
\hline & $X_{m}$ & 0,053 & 0,041 & 0,024 & 0,039 & 0,031 & 0,024 \\
\hline \multirow[t]{2}{*}{ Parâmetros } & $C$ & 10,992 & 14,643 & 28,612 & 45,977 & 38,527 & 55,874 \\
\hline & $\boldsymbol{K}$ & 0,742 & 0,593 & 0,897 & - & - & - \\
\hline $\mathbf{R}^{2}(\%)$ & & 99,3 & 99,3 & 99,4 & 95,4 & 93,9 & 96,6 \\
\hline RQEM (\%) & & 0,69 & 0,77 & 0,98 & 1,81 & 1,41 & 2,45 \\
\hline
\end{tabular}


Pode-se observar na Tabela 1 que os valores obtidos para umidade na monocamada $\left(X_{m}\right)$ se mostraram coerentes, pois diminuíram com o aumento da temperatura. Estes valores são importantes para saber as condições estáveis de conservação do material, pois indicam a quantidade de água que está fortemente adsorvida nos sítios do mesmo.

No trabalho desenvolvido por Lemus et al. (2008) onde estudou-se as isotermas de equilíbrio da alga vermelha Gracilaria, foi obtido o valor de $X_{m}$ igual 0,056 utilizando-se o modelo BET para a temperatura de $40^{\circ} \mathrm{C}$. Já Martins et al. (2004), obteve $X_{m}$ de 0,045 utilizando o modelo $\mathrm{GAB}$ e temperatura de $60^{\circ} \mathrm{C}$ para isotermas de cebola (Allium cepa L.). Estes estudos confirmam a coerência dos valores obtidos no presente trabalho.

Através do coeficiente de determinação $\left(R^{2}\right)$ verificou-se bom ajuste dos modelos aos dados experimentais. A equação de GAB foi escolhida para o cálculo do calor de dessorção em função da umidade por ter apresentado alto coeficiente de determinação $(>0,96)$ e baixo valor para o erro $(<10 \%)$.

A equação de Clausius-Clapeyron mostrou-se adequada para a previsão do calor de dessorção do resíduo da vinificação, com valores de $R^{2}$ superiores a $0,92 \%$. Pode-se analisar na Figura 3 que para ambas as variedades de bagaço, em conteúdos de umidade mais elevados, o valor obtido para o calor de dessorção foi menor, pois quanto mais água está presente no material, menor é a energia de ligação entre as moléculas do sólido e da água, não sendo necessária grande quantidade de calor para promover a remoção desta água.

O mesmo foi observado por Leite et al. (2004), na determinação do calor de dessorção de duas variedades de banana, pois à medida que o seu conteúdo de água diminui, se fez necessário uma maior quantidade de calor para se retirar mais água deste.
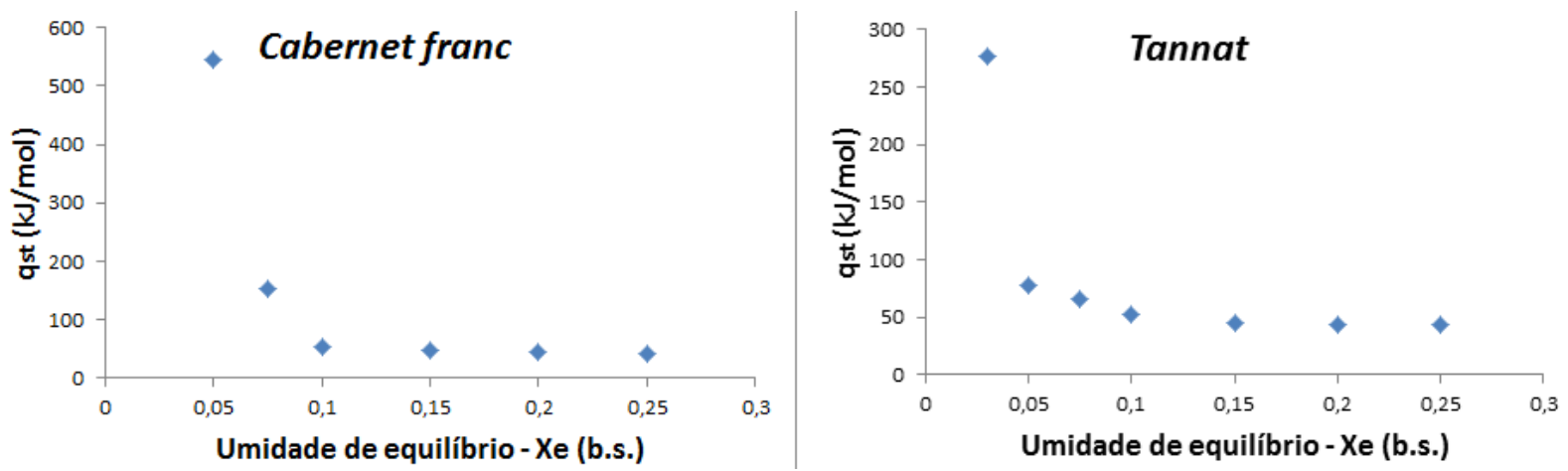

Figura 3 - Calor de dessorção em função da umidade de equilíbrio.

No estudo realizado por Resende et al. (2006) e no desenvolvido por Lemus et al. (2008) para obtenção do calor de dessorção de grãos de feijão e da alga vermelha Gracilaria, respectivamente, foi observado o mesmo comportamento, em que o valor do calor de dessorção, ou seja, da energia necessária para remover água do produto, diminuiu com o aumento do conteúdo de umidade, como já esperado para grãos, legumes, frutas e algas.

\section{CONCLUSÃO}


As isotermas de equilíbrio obtidas pelo método gravimétrico estático nas temperaturas de 50, 60 e $70{ }^{\circ} \mathrm{C}$ apresentaram comportamento característico das isotermas obtidas para produtos alimentícios. Observou-se que o aumento da temperatura influencia na redução da umidade de equilíbrio. $\mathrm{O}$ modelo de $\mathrm{GAB}$ foi o que melhor se ajustou para todas as temperaturas estudadas, com elevado valor para $R^{2}(>0,96)$ e baixo valor de REQM $(<10 \%)$. A equação de Clausius-Clapeyron se mostrou adequada para previsão do calor de dessorção, pois verificou-se que quando há menor quantidade de água no material mais calor é necessário para remover esta água, como já esperado.

\section{NOMENCLATURA}

Símbolo
$\mathrm{C}_{\mathrm{B}}$
$C_{G}$
$K$
$q_{s t}$
$q_{t}$
$R$
$\Delta H_{\text {vap }}$
$T$
$U$
$U R$
$X_{e}$
$X_{m}$

Notação

Constante de modelo

Constante de modelo

Constante de modelo

Calor isostérico líquido de sorção

Calor total de sorção

Constate universal dos gases

Calor de vaporização da água

Temperatura absoluta

Umidade base úmida

Umidade Relativa

Umidade de equilíbrio

Umidade de monocamada
Unidade

$[-]$

$[-]$

$[-]$

$[\mathrm{kJ} / \mathrm{mol}]$

$[\mathrm{kJ} / \mathrm{mol}]$

$\left[\mathrm{kJ} \mathrm{kmol}{ }^{-1} \mathrm{~K}^{-1}\right.$ ]

$[\mathrm{kJ} / \mathrm{mol}]$

[K]

$[-]$

$[-]$

[ $\left.\mathrm{kg} \mathrm{kg}^{-1}\right]$

$\left[\mathrm{kg} \mathrm{kg}^{-1}\right]$

\section{REFERÊNCIAS}

ANDRADE, E. T.; CORREA, P. C.; TEIXEIRA, L. P.; PEREIRA, R. G.; CALOMEN J. de F. Cinética de secagem e qualidade de sementes de feijão. Engev., v. 8, n. 2, p. 83-95, 2006.

ASSOCIATION OF OFFICIAL ANALYTICAL CHEMISTS (A.O.A.C.) (1995), Off. Meth. Anal., ed. 14, 1995.

BHANDARI, B. R.; ADHIKARI, B. P. Water activity in food processing and preservation. In: CHEN, X. D.; MUJUMDAR, A. S. Drying Technologies in Food Processing. Blackwell Publishing Ltd, p. 55-86, 2008.

CAMARGO, U. A.; MAIA, J. D. G.; RITSCHEL, P. Embrapa Uva e Vinho - Novos cultivares brasileiras de uva. Empresa Brasileira de Pesquisa Agropecuária (EMBRAPA Uva e vinho). Editora Embrapa, $1^{\mathrm{a}}$ ed. Bento Gonçalves - RS, 2010.

CAMPOS, A. R. N.; SILVA, F. L. H.; GOMES, J. P.; OLIVEIRA, L. S. C.; OLIVEIRA, M. M. Isotermas de adsorção e calor isostérico da palma forrageira enriquecida proteicamente. Rev. Bra. Eng. Agríc. Amb., Campina Grande, v.13, n.6, p.734-740, 2009. 
GONELI, A. L. D. Variação das propriedades físico-mecânicas e da qualidade da mamona (Ricinus communis L.) durante a secagem e o armazenamento. Universidade Federal de Viçosa (Tese de Doutorado), p. 199, 2008.

IGUAZ, A.; VÍRSEDA, P.; Moisture desorption isotherms of rough rice at high temperatures. J. Food Eng., 79, p. 794-802, 2007.

KUROZAWA, L. E.; EL-AOUAR A. A.; MURR, F. X. Obtenção de isotermas de dessorção de cogumelo in natura e desidratado osmoticamente. Ciência Tecnol. Alime., v. 25, n. 4, p. 828-834, 2005.

LEMUS, R. A.; PÉREZ, M.; ANDRÉS, A.; ROCO, T.; TELlO, C. M.; VEJA, A. Kinetic study of dehydration and desorption isotherms of red alga Gracilaria. LWT - Food Sci. Technol., 41, p. 1592-1599, 2008.

LIMA, E. E. de.; SILVA, A. S'A.; FIGUEIREDO, R. M. F.; QUEIROZ, A. J. M. Estudo das isotermas e calor isostérico de adsorção da farinha da coroa de frade. Rev. Bra. Eng. Agríc. Amb., Campina Grande, v.10, n.2, p. 163-170, 2008.

LUZ, G. R.; SOUSA, L. H. C. D.; JORGE, L. M. M.; PARAÍSO, P. R. Estudo das isotermas de equilíbrio do farelo de soja. Ciência Tecnol. Alime., Campinas, v.26. n.2. p. 408-413, abr.jun. 2006.

MARINOS-KOURIS, D.; MAROULIS, Z. B. Transport Properties in the Drying of Solids. In: MUJUMDAR, A. S. Handbook of Industrial Drying, Taylor \& Francis Group LLC, $3^{\mathrm{a}}$ ed., 2006.

MELLO, L. M. R. Vitinicultura brasileira: panorama e atuação no mercado em 2012. Empresa Brasileira de Pesquisa Agropecuária (EMBRAPA - UVA e vinha). Bento Gonçalves - RS, 2013.

PACHECO, A. C. W.; LUZ, G. R.; JORGE, L. M. M.; PARAÍSO, P. R. Determinação das isotermas de equilíbrio na adsorção da ração de peixe. In: VIII Congresso Brasileiro de Engenharia Química em Iniciação Científica, Uberlândia, Minas Gerais, 2009.

PARK K. J.; BIN A.; BROD, F. P. R. Obtenção das isotermas de sorção e modelagem matemática para a pêra bartlett (pyrus sp.) com e sem desidratação osmótica. Ciência. Tecnol. Alime.,v. 21, n. 1, p.73-77, 2001.

RESENDE, O.; CORRÊA, P. C.; GONELI, A. L. D.; RIBEIRO, D. M. Isotermas e calor isostérico de sorção do feijão. Ciência. Tecnol. Alimen., v. 26, n. 3, p. 626-631, 2006.

SHIEBER, D. F.; STINTZING, C.; CARLE, R. By-produtcs of Plant Food Processing as Sourtve of Functional Compounds-Recent Developments. Trend Food Sci Technol., v. 12, p. 401-413, 2001. 